\title{
UNIOCULAR FIELDS OF FIXATION IN THYROID EYE DISEASE
}

\author{
D. H. W. STEEL, H. B. HOH, M. J. POTTS and R. A. HARRAD \\ Bristol
}

\begin{abstract}
SUMMARY
Immunosuppressive therapy is well established in the treatment of thyroid eye disease (TED). The best response has been observed in those with active (wet phase) disease of short duration. A prospective study was designed to observe the effects of orbital radiotherapy and oral immunosuppression on patients with TED, and to assess whether any pre-treatment parameters were predictive of the outcome. Significant improvements in uniocular fields of fixation (UFOF) and in the Mourits' disease activity scale were seen after treatment. The degree of improvement in UFOF was positively correlated with the level of initial disease activity. The use and technique of UFOF in assessing disease phase and activity are discussed.
\end{abstract}

Thyroid eye disease (TED) is thought to be an autoimmune disease although the exact pathogenesis is still unclear. ${ }^{1}$ The disease can be divided into two phases: an active or wet phase with progressive and changing signs, followed by a 'burnt-out' or dry phase with more stable signs. ${ }^{2}$ The active phase is very variable in both severity and duration, with only a small proportion of patients developing severe ophthalmopathy and the majority developing only mild signs which often resolve without treatment. ${ }^{1}$

Rundle and Wilson ${ }^{2}$ described a curve of disease severity against time. There are a number of immunosuppressive regimes, including oral and intravenous steroids, orbital radiotherapy, azathioprine and cyclosporine. It has generally been observed that the best response to medical treatment has been in those with active disease of recent onset. $^{3}$ Reliable measures of disease activity are needed in order to plot the position of individual patients on 'Rundle's curve' and hence to predict likely disease progression and response to treatment. Mourits et al. ${ }^{5}$ have devised a scheme of clinical criteria for assessing disease activity. This differs

Correspondence to: Mr D. H. W. Steel, Bristol Eye Hospital, Lower Maudlin Street, Bristol BS1 2LX, UK. from the well-established American Graves' Disease Classification System (Werner's) ${ }^{4}$ in that it tries to differentiate active from 'burnt-out' disease. Mourits et al. have suggested that the degree of disease activity, rather than the duration of the ophthalmopathy, is the most important factor in predicting treatment success. ${ }^{5}$

The role of immunosuppressive therapy in severe ophthalmopathy is well established. However, in moderate ophthalmopathy, especially in those with disease of long duration, treatment is far more controversial. $^{3}$

We have been treating patients with early, rapidly progressive, moderate disease, as well as those with severe ophthalmopathy, in an attempt to limit the development of sight-threatening complications, ocular movement restriction and its consequences, as well as cosmetic and symptomatic disabilities.

We have previously reported the use of Short Tau Inversion Recovery (STIR) sequence magnetic resonance imaging (MRI) to assess the degree of disease activity in TED. ${ }^{6,7}$ Analysis of patients with TED has shown that the sign that correlates best with disease severity is ocular movement restriction. ${ }^{8}$ This has also been shown to correlate with muscle size on computed tomography and subsequent development of optic neuropathy. ${ }^{9}$ Increasing restriction of ocular movement and the rate of that change can be related to the presence and degree of disease activity.

\section{AIM}

We designed a prospective study to assess the effect of immunosuppression on various clinical measures of disease activity in TED, and to identify which parameters, if any, were predictive of a successful outcome. As part of the study we used three methods of assessing ocular motility, namely uniocular fields of fixation (UFOF), Hess charts, and fields of binocular single vision (BSV). Hess and BSV, being binocular techniques, measure the degree of ocular incomitance. They therefore confound two variables, 
i.e. the individual ocular movement restriction of right and left eyes, and do not measure the absolute change in ocular motility. However, they do relate closely to patient satisfaction and binocular function.

We have found UFOF to be useful for measuring absolute ocular motility in each eye and as a means of monitoring disease activity. We have used this quantitative assessment of changing activity, along with other signs of activity, such as STIR sequence MRI, to guide medical therapy in three ways:

1. To assess disease activity prior to treatment.

2. To assess the response to treatment, including the response to a short high-dose trial of oral steroids when the phase of disease activity is difficult to ascertain (i.e. the active 'wet' or dry 'burnt out' phase).

3. To assess disease relapse following treatment and to help guide the gradual withdrawal of immunosuppression.

In order to assess the effectiveness of our treatment of active disease, and the correlation of UFOF with disease activity, we collected data from a consecutive series of 20 patients who had orbital radiotherapy and oral immunosuppression for TED.

\section{METHOD}

Between March 1992 and October 1993, 20 patients were treated with orbital radiotherapy for thyroidassociated ophthalmopathy at the Bristol Eye Hospital. Oral steroids (20-30 mg) were given concurrently for approximately 6 weeks in order to control any temporary exacerbation of the eye disease induced by the radiotherapy. The orbital radiotherapy was administered by a modification of the Vancouver technique with a $4 \mathrm{MeV}$ linear accelerator. The dose given was 20 gray in 10 fractions over a 12 day period.

Four patients were excluded from the study due to missing data.

All patients were euthyroid at the time of treatment, previous hyperthyroidism having been managed either medically with carbimazole, surgically or with iodine-131 treatment. Only 1 patient had been euthyroid throughout.

Nine patients had severe ophthalmopathy with optic neuropathy. Three of these had asymmetrical signs. Eight patients had more moderate ophthalmopathy without either optic neuropathy or corneal exposure. Only 1 of these had asymmetrical signs.

The following additional data were collected from the 16 patients before treatment and 4-6 months after treatment: (1) areas of UFOF for each eye, (2) Werner's ophthalmopathy classification grade, and (3) Mourits' disease activity grade for each eye. (The change in ocular motility restriction for the Mourits grade was based on changes in the UFOF pre- and post-dating those used in assessment (1) above.

The UFOF values were plotted using a Goldmann field machine with a III 4E target. A reading correction with a wide aperture lens was used if required. A careful explanation of the procedure was given to the patient and fixation monitored using the central telescope. One eye was occluded during the procedure and the patient instructed to follow the outwardly moving target, without any head movement, until foveal fixation could no longer be continued. The difference between fixating the target and observing it in the patient's peripheral field of vision was explained. Observation of ocular movement via the central telescope was useful in verifying this subjective end-point of peripheral fixation. The procedure was then repeated at approximately $30^{\circ}$ intervals around the field. In the majority of patients the UFOF were plotted by the same observer, and all fields were completed at the same time of day.

\section{Statistical Analysis}

Paired $t$-tests were used to assess changes in UFOF and disease activity following treatment. Multivariable analysis, using the Generalised Linear Interactive Modelling system (GLIM) of the Numerical Algorithms Group (NAG), was used to look for correlation between the change in UFOF area following treatment and (1) initial disease activity and (2) initial UFOF area. Possible confounding effects of the co-variables age and disease duration, as well as of the co-factor gender, were controlled for in the analysis. Inter-eye correlation was included in the analysis using Rossner's intra-class correlation model throughout.

\section{RESULTS}

The age range of the patients studied was 23-67 years with a mean of 55 years. There were 9 men and 7 women. Disease duration ranged from 3 to 72 months with a mean of 21 months. One patient in the study had a blind left eye secondary to trauma. No UFOF were recorded for this eye.

All patients in the study showed an improvement in American classification following immunosuppression, with either a decrease in class or a decrease in grade within a class. All patients showed an enlargement of their UFOF. The mean area before treatment of $5444 \mathrm{~mm}^{2}$ improved to $9124 \mathrm{~mm}^{2}$ after treatment, with a mean improvement of $3680 \mathrm{~mm}^{2}$; this improvement was significant $(r=0.54, p=0.008)$ (Fig. 1).

Mean activity score based on Mourits' schemes before treatment was 4.6 with a range of 1 to 8 . This was reduced significantly after treatment to a mean of 0.5 ( $r=0.84, p<10^{-6}$ (Fig. 2). 


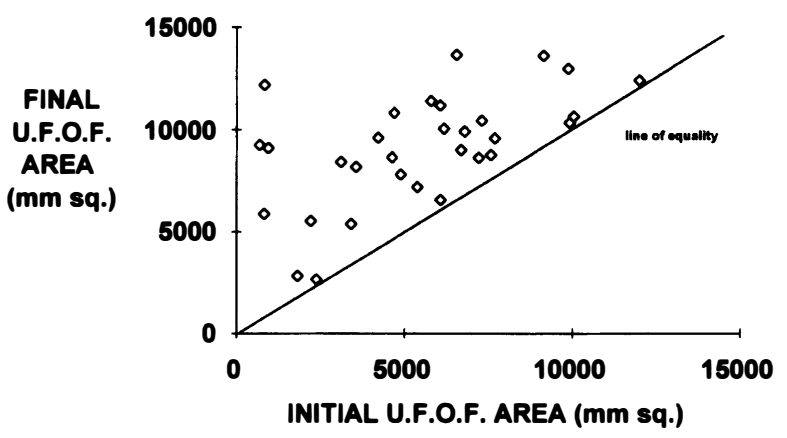

Fig. 1. Final UFOF area (mean $9124 \mathrm{~mm}^{2}$ ) versus initial UFOF area (mean $5444 \mathrm{~mm}^{2}$ ).

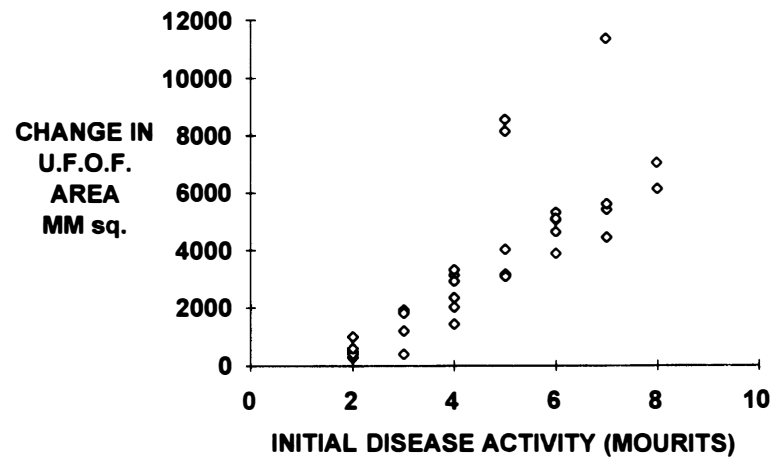

Fig. 3. UFOF change after treatment (mean $3680 \mathrm{~mm}^{2}$ ) versus initial disease activity.

In 4 patients disease activity increased to near preradiotherapy levels as oral steroids were withdrawn after radiotherapy. In these patients azathioprine (100-150 mg per day) was added due to advancing signs and symptoms and significantly high activity scores ( $>4)$. This led to an improvement in activity score and UFOF area compared with pre-treatment levels. Initial activity score in this group ranged from 4 to 6, with UFOF improvement ranging from $8560 \mathrm{~mm}^{2}$ to $1200 \mathrm{~mm}^{2}$. In 1 patient the activity score did not reduce following treatment, being unchanged at 2. Further immunosuppression was not added in this case because of the low activity score.

Initial disease activity was highly correlated with the change in UFOF after treatment (partial correlation coefficient (p.c.c.) $r=0.57, \quad p<0.001$ ) (Fig. 3). All patients, except one, had a disease activity score of one or less after treatment and the change in UFOF was also strongly correlated with the change in disease activity with treatment (p.c.c. $r$ $=0.52, p=0.001)$. Disease duration was not correlated with the change in UFOF (p.c.c.) $r=15.7$ $\times 10^{-4}, p=0.67$ ) (Fig. 4). The correlation between initial UFOF area and changes in UFOF area following treatment achieved significance when initial disease activity was included in the analysis (p.c.c. $r=0.5, p<0.001$ ), i.e. UFOF alone without other indicators of disease activity was not correlated with changes in UFOF. Youth (p.c.c. $r=0.04, p=$

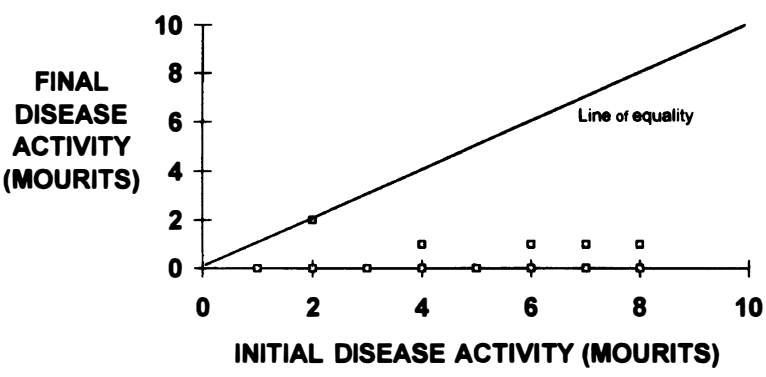

Fig. 2 Final disease activity (mean 0.5 Mourits) versus initial disease activity (mean 4.6 Mourits).

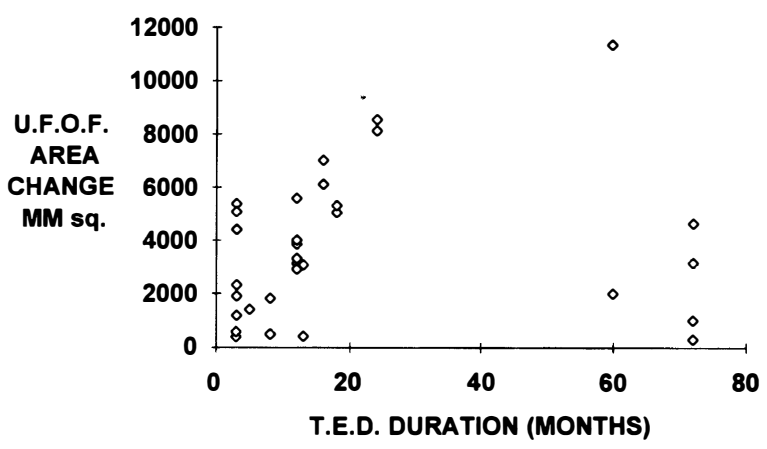

Fig. 4. UFOF change after treatment versus duration of $T E D$.

$0.0041)$ and male sex (p.c.c. $r=0.19, p=0.00004$ ) were positively correlated with treatment success.

To estimate the effect of inter- and intraobserver variation in replicate measurements of UFOF, 6 patients were recalled and UFOF replotted. For each patient UFOF were plotted twice by one examiner, and then repeated by an alternative examiner. In addition, two further UFOF were recorded without fixation monitoring, the end-point of peripheral fixation during these fields being assessed only by the patients' verbal responses. Analysis of these UFOF (after logarithmic transformation) showed the following repeatability coefficients (this was defined as two times the standard deviation of the differences, as adopted by the British Standards Institution $\left.^{10}\right)$ :

(A) Two consecutive fields, same examiner, fixation monitored: $8.9 \%$.

(B) Two consecutive fields, different examiners, fixation monitored: $13.9 \%$.

(C) Two consecutive fields, same examiner, no fixation monitoring: $20.1 \%$.

Thus serial examinations by one examiner, and fixation monitoring to help localise the end-point of peripheral fixation, improve the repeatability of UFOF. 


\section{DISCUSSION}

Changes in UFOF reflect changes in disease activity in thyroid eye disease. Most of the patients in the study showed a significant improvement in UFOF and disease activity following radiotherapy with or without oral immunosuppression. In common with other studies, ${ }^{11,12}$ there were few adverse affects of radiotherapy in the short follow-up period. Although the study was uncontrolled, the treatment appeared to be very effective, encouraging us to treat patients with early but progressive ophthalmopathy in an attempt to prevent the development of more severe complications and disabilities.

Of the 5 patients in whom disease activity remained high after radiotherapy, 4 were successfully treated with azathioprine. One other patient with a low initial activity, which did not improve with radiotherapy, was not treated with further immunosuppression.

We find the estimated activity of the autoimmune orbitopathy to be a better indicator of disease response to immunosuppression than is disease duration alone. Some cases of ophthalmopathy appear to have a prolonged phase of low activity, with mild symptoms and signs, before becoming more active with a concomitant increase in the severity of the signs. The results are in accordance with our clinical experience in that the more active a patient's disease, the greater the response expected from immunosuppression.

The technique of UFOF can be difficult. Careful patient explanation and encouragement are important. Our small study of replicate field testing shows that fixation monitoring and serial examinations by one examiner increase the repeatability of the test. We believe that these two factors have made UFOF a more reliable test for use in TED, in contrast to others who have reported problems with the technique. ${ }^{13}$

It should be stressed, however, that we do not find solitary measures of UFOF particularly useful in individual patients. It is the change in UFOF that is important. This is because of two factors. Firstly there is a high inter-individual variability in UFOF depending on factors such as facial structure and age as well as ocular motility restriction. Secondly, single UFOF provide no information on disease activity and patients with long-standing inactive disease can have very constricted fields. Initial UFOF area was only predictive of improvement in UFOF with treatment if activity was taken into account. Hence a constricted UFOF is not predictive of a successful response to treatment unless disease activity is high.

In conclusion, changes in UFOF appear to reflect changes in disease activity in TED, and can be used to help select patients suitable for immunosuppressive therapy. UFOF can also help monitor the response to oral immunosuppressants and guide their withdrawal following radiotherapy, as natural disease activity decreases. In cases where it is uncertain whether the disease is active or 'burntout', a short high-dose trial of steroids while monitoring UFOF can help assess the likely benefit of orbital immunosuppressive therapy.

We would like to thank Mr J. Sparrow for his advice on the statistical analysis, Miss L. Newcombe and the staff of the orthoptic department, and Mrs G. Bennerson of the photography department.

Key words: Orbital radiotherapy, Thyroid eye disease, Uniocular fields of fixation.

\section{REFERENCES}

1. Bahn RS, Heufelder AE. Pathogenesis of Graves' ophthalmopathy. N Engl J Med 1993;329:1468-75.

2. Rundle FF, Wilson CW. Development and course of exophthalmos and ophthalmoplegia in Graves' disease with special reference to the effect of thyroidectomy. Clin Sci 1944;5:177-94.

3. Kendal-Taylor P. The management of Graves' ophthalmopathy. Clin Endocrinol 1989;31:747-56.

4. Werner SC. Modification of the classification of the eye changes of Graves' disease: recommendations of the ad hoc committee of the American Thyroid Association. J Clin Endocrinol Metab 1977;44:203-4.

5. Mourits MP, Koornneef LK, Wiersinga WM, et al. Clinical criteria for the assessment of disease activity in Graves' ophthalmopathy: a novel approach. $\mathrm{Br} \mathrm{J}$ Ophthalmol 1989;73:639-44.

6. Laitt RD, Hoh B, Wakeley CJ, Kabala J, Harrad R, Potts M, Goddard P. The value of the STIR sequence in magnetic resonance imaging of thyroid eye disease. $\mathrm{Br}$ J Radiol 1994;67:244-7.

7. Hoh B, Laitt R, Wakeley C, Kabala J, Harrad R, Potts $M$, Goddard P. The value of STIR sequence in magnetic resonance imaging of thryoid eye disease. Eye 1994;8:506-10.

8. Feldon SC, Muramatsu S, Weiner J. Clinical classification of Graves' ophthalmopathy: identification of risk factors for optic neuropathy. Arch Ophthalmol 1984; 102:1469-1472.

9. Hallin ES, Feldon SE. Graves' ophthalmopathy: correlation of clinical signs with measures derived from computed tomography. Br J Ophthalmol 1988; $72: 678-82$

10. Bland JM, Altman DG. Statistical methods for assessing agreement between two methods of clinical measurement. Lancet 1986;11:307-10.

11. Kazim M, Trokel S, Moore S. Treatment of acute Graves' orbitopathy. Ophthalmology 1991;98:1443-8.

12. Peterson IA, Kriss JP, McDougal IR, Donaldson IR. Prognostic factors in the radiotherapy of Graves' ophthalmopathy. Int $\mathrm{J}$ Radiat Oncol Biol Phys 1990;19:259-64.

13. McCarry B, Kousoulides L, Aylward GW, et al. Uniocular fields of fixation in thyroid eye disease. In: Kaufmann H, editor. Transactions of 21st Meeting of the European Strabismological Association, Salzburg, June 1993: 391-6. 